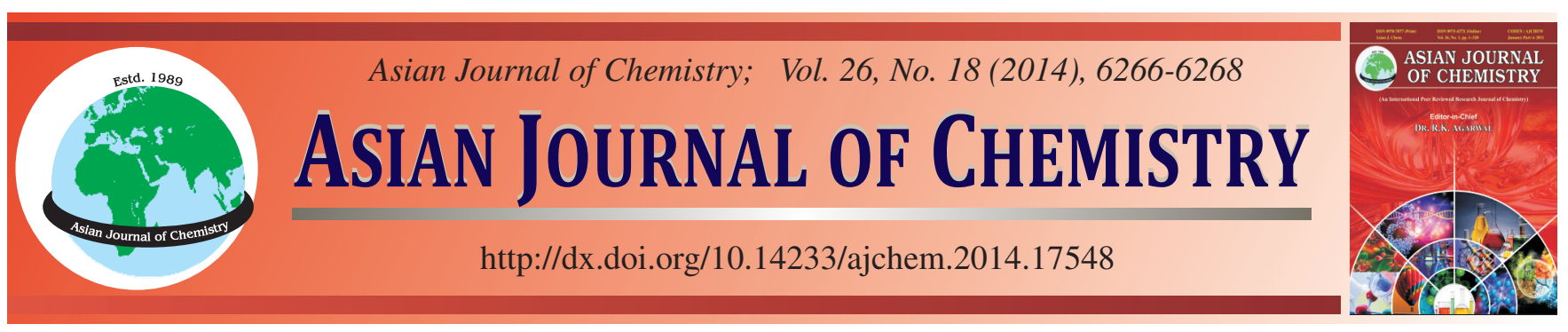

\title{
Quality Analysis of Five Bioactive Compounds in Mentha haplocalyx Extracts by High Performance Liquid Chromatography
}

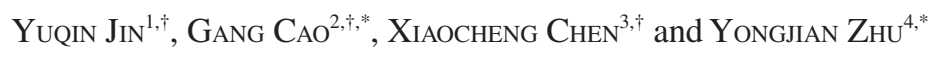

${ }^{1}$ The 2nd Affiliated Hospital, Wenzhou Medical University, Wenzhou, P.R. China

${ }^{2}$ Research Center of TCM Processing Technology, Zhejiang Chinese Medical University, Hangzhou, P.R. China

${ }^{3}$ The First Affiliated Hospital, Wenzhou Medical University, Wenzhou, P.R. China

${ }^{4}$ Department of Neurosurgery, Second Affiliated Hospital of Zhejiang University School of Medicine, Hangzhou, P.R. China

$\uparrow$ These authors contribute equally to this work

*Corresponding authors: Tel/Fax: +86 571 87195895; E-mail: caogang33@163.com

Fax: +86 571 87784785; Tel: +86 571 87784787; E-mail: yongjian_zhu@zju.edu.cn

Received: 4 March 2014;

Accepted: 21 April 2014;

Published online: 1 September 2014;

AJC-15895

\begin{abstract}
Mentha haplocalyx is derived from the aerial part of Mentha haplocalyx Brip. Caffeic acid, hesperidin, rosmarinic acid, buddleoside and diosmetin are active constituent of Mentha haplocalyx used in many traditional Chinese medicines. This paper describes a sensitive and specific assay for the determination of five bioactive compounds in Mentha haplocalyx extracts. In this paper, the five components were separated on an Agilent Zorbax Eclipse XDB- $\mathrm{C}_{18}$ column $(250 \times 4.6 \mathrm{~mm}, 5 \mu \mathrm{m})$ and detected by diode array detector (DAD). Mobile phase was composed of (A) aqueous phosphoric acid $(0.5 \%, v / v)$ and (B) acetonitrile using a gradient elution. Analytes were performed at $30{ }^{\circ} \mathrm{C}$ with a flow rate of $1 \mathrm{~mL} / \mathrm{min}$ and UV detection at $340 \mathrm{~nm}$. All calibration curves showed good linear regression $\left(\mathrm{r}^{2} \geq 0.9999\right)$ within tested ranges. The LOD and LOQ were $0.31-5.93$ and $2.98-57 \mu \mathrm{g} / \mathrm{mL}$, respectively. Overall intra-day and inter-day variations were less than $2.19 \%$ and th e average recoveries were $98.23-100.04 \%$ for the analytes. This newly established method is validated as simple, precise and accurate. It can be used as a valid analytical method for intrinsic quality control of Mentha haplocalyx. In conclusion, the proposed method would be sensitive enough and reliable for comprehensive quality control for clinical use and modernization of traditional Chinese medicines.
\end{abstract}

Keywords: HPLC-DAD, Mentha haplocalyx, Quality control, Simultaneous determination, Traditional Chinese medicines.

\section{INTRODUCTION}

Traditional Chinese medicines (TCMs) has a robust history with roots dating back thousands of years for the medicinal practice in China and some East Asian countries ${ }^{1-3}$. Mentha haplocalyx, a commonly used traditional Chinese medicines called Bohe in Chinese, is derived from the the aerial part of Mentha haplocalyx Brip ${ }^{4}$. It is widely distributed in Jiangsu, Anhui, Jiangxi and Zhejiang provinces of China, is not only used as popular vegetable but also widely used in the treatment of nerve center, breath, procreation and digestive systems in China ${ }^{5,6}$. To understand the mechanisms involved in these beneficial effects, a great deal of scientific efforts have been contributed to isolate and identify the active components in various Mentha haplocalyx samples. Volatile components, flavonoids and polyphenolic acids are the main components in Mentha haplocalyx samples, especially volatile compounds have been considered the main players in these benefits on the human health ${ }^{7,8}$. Our preliminary experiments showed that
Mentha haplocalyx contained significant amounts of polyphenolic compounds and flavonoids, which exhibited considerable radical scavenging activity in DPPH assay. There are reports on the determination of multiple bioactive components in traditional Chinese medicines, such as determination of gallic acid, 5-hydroxymethylfurfural (5-HMF), morroniside, sweroside, loganin and cornuside in Fructus Corni (9) and on the analysis of paeoniflorin, laetrile Calycosin-7-O- $\beta-\mathrm{D}-$ glucoside, ononin, calycosin and formononetin in Buyang Huanwu decoction ${ }^{10}$. However, the multicomponent analysis of traditional Chinese medicines is not easy due to the complexity of their components. To our best of knowledge, previous studies on Mentha haplocalyx are mainly focused on volatile components ${ }^{11}$, but there are no reports on the quantitative determination of the multiple components in Mentha haplocalyx. In this study, a RP-HPLC-DAD method was developed for simultaneous determination five major active components in Mentha haplocalyx distributed in China. 


\section{EXPERIMENTAL}

Mentha haplocalyx was collected from three suppliers (Jiangsu, Zhejiang, Anhui in China) and identified by Prof. Baochang Cai in Zhejiang Chinese Medical University. Reference standards of caffeic acid and hesperidin were purchased from the National Institute for the Control of Pharmaceutical and Biological Products (Beijing, China). Rosmarinic acid, buddleoside and diosmetin were purchased from Shanghai Tongtian Biotechnology Co. Ltd. (Shanghai, China). The purity for each standard compound was greater than $98 \%$ by HPLC analysis. The structures of these five compounds were shown in Fig. 1. All reagents with high grade were obtained from others. Milli-Q water (Millipore, Bedford, MA, USA) was used throughout the study.

Preparation of sample solutions: The powder of Mentha haplocalyx samples quantitatively ( $1 \mathrm{~g}$ ) transferred into dark brown calibrated flasks and extracted with $50 \mathrm{~mL}$ of methanol in an ultrasonic bath for $0.5 \mathrm{~h}$ and cooled at room temperature; methanol was added to compensate for the lost weight. The solution was filtered through a $0.45 \mu \mathrm{m}$ membrane filter before subjecting $10 \mu \mathrm{L}$ to HPLC analysis.

Preparation of standard solutions and calibration curve: Each reference standard was dried and accurately weighed, then dissolved in methanol and diluted to appropriate concentration, respectively. A mixed stock solution of standards, containing caffeic acid $(0.059 \mathrm{mg} / \mathrm{mL})$, hesperidin $(1.140 \mathrm{mg} / \mathrm{mL})$, rosmarinic acid $(0.580 \mathrm{mg} / \mathrm{mL})$, buddleoside $(0.620 \mathrm{mg} / \mathrm{mL})$ and diosmetin $(0.059 \mathrm{mg} / \mathrm{mL})$, was finally prepared. The standard stock and working solutions were all prepared in calibrated flasks and stored at $4{ }^{\circ} \mathrm{C}$. All calibration curves were constructed from peak areas of the reference standards versus their concentrations. The solutions were filtered through a $0.45 \mu \mathrm{m}$ membrane prior to injection.

Chromatographis analysis: Analyses were performed using Agilent 1200 HPLC system (Agilent Technologies, Palo Alto, CA, USA) with diode array detector. Detection wave-

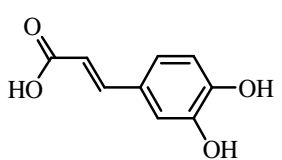

Caffeic acid<smiles>O=C(/C=C\c1ccc(O)c(O)c1)OCC(C(=O)O)c1ccc(O)c(O)c1</smiles>

Rosmarinic acid<smiles>COc1ccc(C2CC(=O)c3c(O)cc(O)cc3O2)cc1O</smiles>

Diosmetin

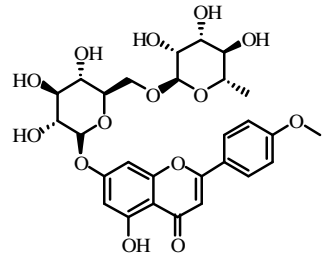

Hesperidin

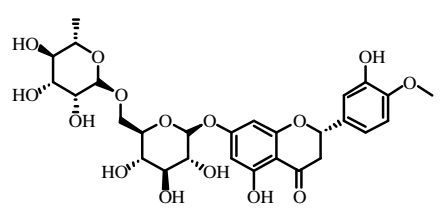

Buddleoside
Fig. 1. Chemical structures of the five active components in Mentha hapioealyx lengths were set at $340 \mathrm{~nm}$. An Agilent Zorbax Extend $\mathrm{C}_{18}$ $(250 \times 4.6 \mathrm{~mm}, 5 \mu \mathrm{m})$ was used with a flow rate of $1 \mathrm{~mL} / \mathrm{min}$. The injection volume was $10 \mu \mathrm{L}$ and the column temperature was maintained at $30^{\circ} \mathrm{C}$. Mobile phase was composed of (A) aqueous phosphoric acid (0.5\%, v/v) and (B) acetonitrile using a gradient elution of $0-80 \mathrm{~min}, 10-45 \% \mathrm{~B}$.

\section{RESULTS AND DISCUSSION}

Optimization of HPLC chromatography conditions: The optimization of experimental conditions was guided by the requirement to obtain chromatograms with better resolution of adjacent peaks, especially when numerous similar components were analyzed. The aim of this study was to develop a HPLC method using diode array detector detection for simultaneous determination of caffeic acid, hesperidin, rosmarinic acid, buddleoside and diosmetin in Mentha haplocalyx samples. Different mobile phase compositions were tested: (1) watermethanol; (2) water-acetonitrile; (3) aqueous phosphoric acid $(0.2 \%, \mathrm{v} / \mathrm{v})$-acetonitrile; (4) aqueous ammonium acetate $(0.1$ $\%, \mathrm{v} / \mathrm{v})$-acetonitrile; (5) aqueous phosphoric acid (0.5\%, v/v)acetonitrile. As a result, the combination of aqueous phosphoric acid $(0.5 \%, \mathrm{v} / \mathrm{v})$-acetonitrile for mobile phase was the best for separation. Furthermore, other chromatographic variables were also optimized, including analytical columns (Hanbon Hedera ODS-2, Hanbon Lichrospher $\mathrm{C}_{18}$ and Agilent Zorbax Eclipse XDB- $\left.\mathrm{C}_{18}\right)$, the column temperatures $\left(20,25\right.$ and $\left.30{ }^{\circ} \mathrm{C}\right)$ and the flow rates $(0.5$ and $1.0 \mathrm{~mL} / \mathrm{min})$. Eventually, the optimal separation was achieved on an Agilent Zorbax Extend $\mathrm{C}_{18}$ column $(250 \times 4.6 \mathrm{~mm}, 5 \mu \mathrm{m})$ at a column temperature of $30{ }^{\circ} \mathrm{C}$ with a flow rate of $1 \mathrm{~mL} / \mathrm{min}$. Fig. 2 showed the typical separation of a standard mixture (A) and Mentha haplocalyx extracts (B) obtained under the above optimized HPLC conditions.
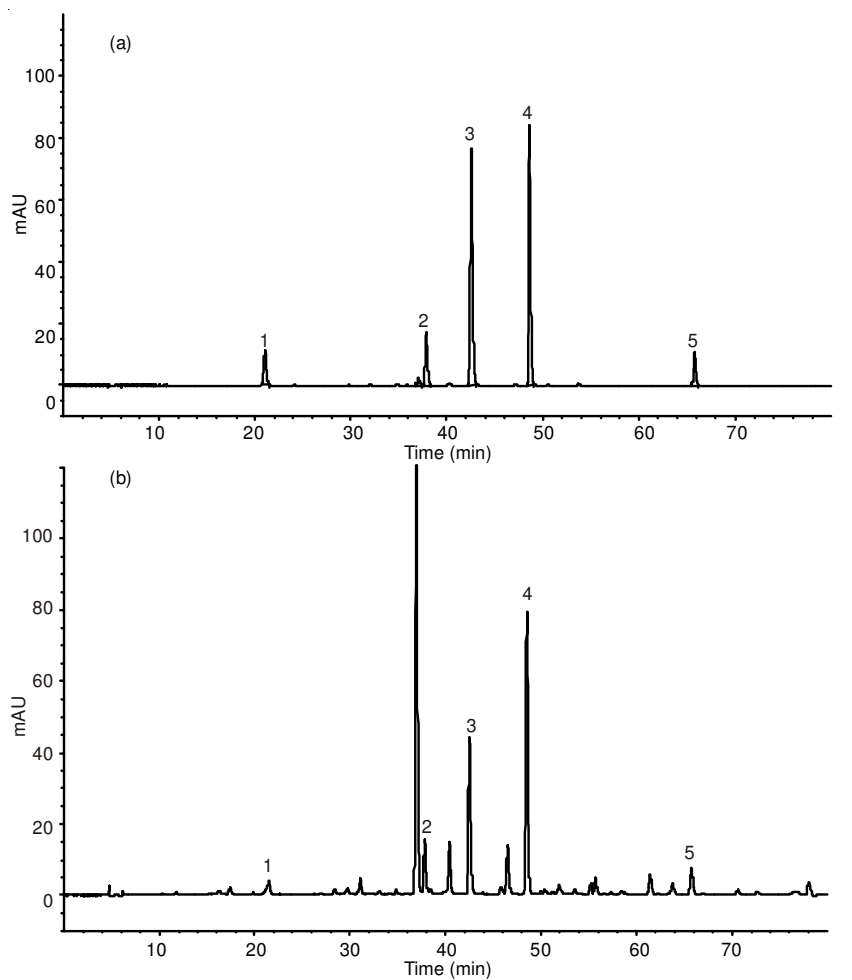

Fig. 2. Typical chromatograms of reference compounds (A), Mentha hapioealyx sample (B). (1) caffeic acid; (2) hesperidin; (3) rosmarinic acid; (4) buddleoside and (5) diosmetin 
TABLE-1

CONTENTS OF THE FIVE COMPONENTS IN Mentha hapioealyx (mg/g \pm S.D., $\mathrm{n}=5$ )

\begin{tabular}{ccccccc}
\hline Samples & Suppliers & Caffeic acid & Hesperidin & Rosmarinic acid & Buddleoside & Diosmetin \\
\hline Mentha & Jiangsu & $0.62 \pm 0.01$ & $24.33 \pm 0.10$ & $8.26 \pm 0.06$ & $14.29 \pm 0.05$ & $1.08 \pm 0.01$ \\
hapioealyx & Zhejiang & $0.59 \pm 0.01$ & $24.21 \pm 0.36$ & $8.08 \pm 0.01$ & $14.27 \pm 0.03$ & $1.01 \pm 0.01$ \\
& Anhui & $0.59 \pm 0.01$ & $24.03 \pm 0.05$ & $8.04 \pm 0.04$ & $14.05 \pm 0.04$ & $1.06 \pm 0.04$ \\
\hline
\end{tabular}

Linearity: The linearity calibration curves were constructed by six concentration assays of each reference compound in triplicate. An aliquot $(10 \mu \mathrm{L})$ of each standard solution was subjected to HPLC analysis. The regression equations were calculated in the form of $y=a x+b$, where $y$ and $x$ were the values of the peak area and concentration of each reference compound, respectively. The limit of detection and quantification under the chromatographic conditions were determined by injecting a series of standard solutions until the signal-to-noise $(\mathrm{S} / \mathrm{N})$ ratio for each compound was 3 for LOD (limit of detection) and 10 for LOQ (limit of quantification). The regression equations (linear ranges) were $\mathrm{y}=6.6137 \mathrm{x}+1.8392(2.98-59.6 \mu \mathrm{g} / \mathrm{mL}$, caffeic acid), $y=0.45 \mathrm{x}+4.9157(57-1140 \mu \mathrm{g} / \mathrm{mL}$, hesperidin), $\mathrm{y}=4.0845 \mathrm{x}+8.611(29-580 \mu \mathrm{g} / \mathrm{mL}$, rosmarinic acid $), \mathrm{y}=3.871 \mathrm{x}$ + $9.0942(31-620 \mu \mathrm{g} / \mathrm{mL}$, buddleoside $), \mathrm{y}=6.9526 \mathrm{x}-1.1901$ (3.12-62.4 $\mu \mathrm{g} / \mathrm{mL}$, diosmetin). All the marker substances showed good linearity $\left(r^{2} \geq 0.9999\right)$. The LOD and LOQ of the five analytes were $0.31-5.93 \mathrm{mg} / \mathrm{mL}$ and $2.98-57 \mu \mathrm{g} / \mathrm{mL}$, respectively.

Precision, repeatability and stability: The intra-day and inter-day precision were determined by analyzing calibration samples during a single day and on three different days, respectively. The intra-day variation was determined by analyzing the six replicates on the same day and inter-day variation was determined on three consecutive days. The relative standard deviation (RSD) was taken as a measure of precision and overall intra-day and inter-day variations were less than $2.19 \%$. To further evaluate the repeatability of the developed assay, Mentha haplocalyx was analyzed in six replicates as described above. The contents of five compounds in Mentha haplocalyx extracts were calculated from the corresponding calibration curves. The relative standard deviations were taken as measurements of repeatability. Stability was tested with Mentha haplocalyx extracts at room temperature and analyzed at $0 \mathrm{~h}, 2 \mathrm{~h}, 4 \mathrm{~h}, 8 \mathrm{~h}$, $12 \mathrm{~h}, 24$ and $48 \mathrm{~h}$ within 2 days, respectively. The relative standard deviations of repeatability test and stability were not more than $2.89 \%$ for all analytes.

Accuracy: Accuracy was determined by the recovery test. An appropriate amount of Mentha haplocalyx powder was weighed and spiked with known amount of each standard compound. They were then treated and analyzed as described above. Each sample was analyzed in six replicates. The total amount of each analyte was calculated from the corresponding calibration curve.

$$
\begin{gathered}
\text { Recovery }(\%)=\left(\text { Amount }_{\text {determined }}-\text { Amount }_{\text {original }}\right) / \\
\text { Amount }_{\text {spiked }} \times 100 \%
\end{gathered}
$$

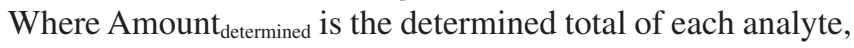
Amount $_{\text {original }}$ is the original amount of each analyte in Mentha haplocalyx samples measured and Amount spiked $_{\text {is }}$ the spiked amount of each analyte. For comparison, an unspiked sample was prepared and analyzed simultaneously. Mean recoveries of the compounds were 98.23-100.04\%, with relative standard deviation values ranging from 1.86 to $2.05 \%(n=6)$.
Sample analysis: The contents of caffeic acid, hesperidin, rosmarinic acid, buddleoside and diosmetin in Mentha haplocalyx samples were determined by the proposed HPLC method under the conditions described previously. The contents were calculated and summarized $(n=6)$ in Table-1. According to the quantitative analysis results, we noticed that the total contents of five compounds varied slightly in the same type of samples from different suppliers, which might be due to the differences in soils and climates in each region. Thus it is necessary to control the main active components in Mentha haplocalyx by good agricultural practice and the norm of Chinese medicinal materials processing.

\section{Conclusion}

An HPLC-DAD method was first developed to simultaneously determine caffeic acid, hesperidin, rosmarinic acid, buddleoside and diosmetin in Mentha haplocalyx samples with an economical mobile phase. All Mentha haplocalyx samples contain large amounts of hesperidin,rosmarinic acid and diosmetin. The contents of caffeic acid, hesperidin,rosmarinic acid, buddleoside and diosmetin are related to quality of Mentha haplocalyx manufacturing. This newly established method is validated as simple, precise and accurate. It can be used as a valid analytical method for intrinsic quality control of Mentha haplocalyx. In conclusion, the proposed method would be sensitive enough and reliable for comprehensive quality control for clinical use and modernization of traditional Chinese medicines.

\section{ACKNOWLEDGEMENTS}

This work was financially supported by the Project of Science and Technology for Chinese Medicine of Zhejiang Province, China (no. 2013KYB183), and the Science and Technology Project of Hangzhou, China (nos 20130533B68 and 20131813A23).

\section{REFERENCES}

1. X.D. Wen, L.W. Qi, J. Chen, Y. Song, L. Yi, X.W. Yang and P. Li, J. Chromatogr. B, 852, 598 (2007).

2. Z.M. Qian, S.J. Qin, L. Yi, H.J. Li, P. Li and X.D. Wen, Biomed. Chromatogr., 22, 202 (2008).

3. W.F. Du, H. Cai, M.Y. Wang, X. Ding, H. Yang and B.C. Cai, J. Pharm. Biomed. Anal., 48, 194 (2008).

4. Y. Su, C.Z. Wang and Y.L. Guo, Acta Chim. Sin., 67, 546 (2009).

5. C.Y. Liang, W.L. Li, H.Q. Zhang and B.R. Ren, Chin. Wild Plant Resour., 22, 9 (2003)

6. Y. Liu, Y.H. Zhang and R.B. Shi, China J. Chin. Mater. Med., 30, 1086 (2005).

7. G.M. She, C. Xu, B. Liu and R.B. Shi, J. Food Sci., 75, 359 (2010).

8. Y.H. Zhang, Y. Liu and J. Hu, Chin. Tradit. Herbal Drugs, 37, 512 (2006).

9. R. Lin, J. Zhang, Z. Hu, G. Yang, Q. Lin, X. Song, J. Li and L. Zhang, Anal. Lett., 43, 2905 (2010).

10. C.G. Lu, C.Y. Liang and W.L. Li, Anhui Agric. Sci., 36, 400 (2008).

11. Q.Y. Shan, G. Cao, H. Cai, X.D. Cong and B.C. Cai, J. Chromatogr. A, 1264, 13 (2012). 